\title{
Linx
}

Revue des linguistes de l'université Paris X Nanterre

$80 \mid 2020$

L'héritage de Jean Dubois et Françoise Dubois-Charlier

\section{Liste des publications de Françoise Dubois-Charlier}

\section{(2) OpenEdition}

1 Journals

Édition électronique

URL : http://journals.openedition.org/linx/6622

DOI : 10.4000/linx.6622

ISSN : 2118-9692

Éditeur

Presses universitaires de Paris Nanterre

Référence électronique

"Liste des publications de Françoise Dubois-Charlier », Linx [En ligne], 80 | 2020, mis en ligne le 10 juillet 2020, consulté le 05 août 2020. URL : http://journals.openedition.org/linx/6622 ; DOI : https:// doi.org/10.4000/linx.6622

Ce document a été généré automatiquement le 5 août 2020

Département de Sciences du langage, Université Paris Ouest 


\section{Liste des publications de Françoise Dubois-Charlier}

\section{Thèse et articles}

1 Dubois-Charlier, F. (1970). Etude neurolinguistique de l'alexie " pure ", contribution historique et analyse neurolinguistique d'un groupe de 14 alexiques. Thèse de $3^{\text {ème }}$ cycle : psychologie, Paris 8.

2 Dubois-Charlier, F. (1970). Eléments de linguistique anglaise: syntaxe. Collection «Langue et Langage », Paris : Larousse Dubois-Charlier, F. (1971). Eléments de linguistique anglaise: la phrase complexe et les nominalisations. Paris : Larousse

Dubois-Charlier, F. (1971). «A neurolinguistic approach to the problems of pure alexia ", in Journal de Psychologie Normale et Pathologique, Vol. 68 (1), Jan, 1971, pp. 39-67. Presses Universitaires de France.

Dubois-Charlier, F. (1972). "A note on alexia », in International Journal of Mental Health, 10/1/1972, Vol. 1, Issue 3, pp. 11-13. International Arts and Sciences Press Dubois-Charlier, F. (1972). "A propos de l'alexie pure", in Langages, $\mathrm{n}^{\circ}$ 25, Neurolinguistique et neuropsychologie, pp. 76-94.

7 Dubois-Charlier, F. (1972). «La sémantique générative: une nouvelle théorie linguistique? », in Langages, $\mathrm{n}^{\circ} 27, \mathrm{pp}$. 5-77.

8 Dubois-Charlier, F. (1975). "Avant-propos: Les premiers articles de Fillmore", in Langages, $\mathrm{n}^{\circ} 38$, pp. 3-17.

Dubois-Charlier, F. (1976). «Les analyses neuropsychologiques de l'alexie : 1838-1969 », in Langages, $\mathrm{n}^{\circ} 44$. Les troubles de la lecture : l'alexie, pp. 20-62.

Dubois-Charlier, F. (1999). "Les relatives en américain contemporain », in ANGLOPHONIA/Sigma 6, pp. 61-112.

Dubois-Charlier, F. (2001). "A propos d'énoncés en there is/are », in De la syntaxe à la narratologie énonciative, pp. 57-80. Paris : Ophrys. 
Dubois-Charlier, F. (2001). "Complément de verbe, de proposition, de phrase, d'énoncé ", in Travaux, 17, Cercle de Linguistique d'Aix-en-Provence, pp. 33-50.

(http://cat.inist.fr/?aModele=afficheN\&cpsidt=14213128)

Dubois-Charlier, F. (2005). «A propos de certaines locutions en français », in BalibarMrabti, A. (éd), Le semi-figement. LINX, $n^{\circ}$ 53, pp. 55-70.

Dubois-Charlier, F. (2008). « Du mot non simple au texte non simple» in B. Kaltz (éd), Regards croisés sur les mots non simples, pp. 151-163. Lyon: ENS Editions, Collection Langages.

\section{Ouvrages en collaboration}

\section{II.1. Ouvrages en collaboration avec Jean Dubois}

Dubois, J. \& F. Dubois-Charlier (1970). Eléments de linguistique française. Paris : Larousse, 295 pages.

Dubois, J. \& F. Dubois-Charlier (1978). Dictionnaire du français langue étrangère Niveau 1. Paris : Larousse, 910 pages.

Dubois, J. \& F. Dubois-Charlier (1979). Dictionnaire du français langue étrangère Niveau 2. Paris : Larousse, 1088 pages.

Dubois, J. \& F. Dubois-Charlier (1982). Larousse de l'orthographe. Paris: Larousse, 176 pages, devenu Nouveau Larousse de l'orthographe en 1994.

Dubois, J., F. Dubois-Charlier et J.P. Mevel, (1993). Dictionnaire de poche de la langue française. Paris : Hachette, 620 pages.

Dubois, J. \& F. Dubois-Charlier (1993). Dictionnaire électronique des verbes français (LVF), remanié en 1994, puis en 2013, 25610 entrées. Téléchargeable sur :

a) https://www.modyco.fr/fr/base-documentaire/ressources/jean-dubois.html (version Excel)

b) http://rali.iro.umontreal.ca/rali/?q=fr/versions-informatisees-lvf-dem (version JOSON)

Dubois, J. \& F. Dubois-Charlier (1994). Les verbes français (d'après le «Dictionnaire Electronique des Verbes »). Paris : Larousse, 458 pages. Téléchargeable sur :

https://www.modyco.fr/fr/base-documentaire/ressources/jean-dubois.html

Dubois, J. \& F. Dubois-Charlier (1995). Dictionnaire électronique des affixes (préfixes et suffixes). 4290 entrées, Aix-en-Provence.

Dubois, J. \& F. Dubois-Charlier (1999). La dérivation suffixale. Paris : Nathan, 320 pages.

Dubois, J. \& F. Dubois-Charlier (2000). Dictionnaire des suffixes en français. Aix-enProvence, 865 pages. Téléchargeable prochainement sur :

https://www.modyco.fr/fr/base-documentaire/ressources/jean-dubois.html

Dubois, J. \& F. Dubois-Charlier (2001). Composition et préfixation. Aix-en-Provence, 312 pages. Téléchargeable prochainement sur :

https://www.modyco.fr/fr/base-documentaire/ressources/jean-dubois.html

Dubois, J. \& F. Dubois-Charlier (2002). Structures verbales. Aix-en-Provence, 303 pages.

Dubois, J. \& F. Dubois-Charlier (2004). Locutions en français. Aix-en-Provence, 414 pages. Téléchargeable prochainement sur : 
https://www.modyco.fr/fr/base-documentaire/ressources/jean-dubois.html JOSON) 79-92. romanes.

Dubois, J. \& F. Dubois-Charlier (2005). Adjectifs en français. Aix-en-Provence, 303 pages.

Dubois, J., Dubois-Charlier, F. et Mitterand, H. (2005). Dictionnaire étymologique et historique du français, refonte totale du Nouveau Dictionnaire étymologique (encadrés, index alphabétique, etc.). Paris : Larousse.

Dubois, J., Dubois-Charlier, F. (2006). Le nombre en français. Aix-en-Provence, 207 pages. Repris dans la collection Collection Poiein, dirigée par Antoinette Balibar, 2008, Amiens : E. M. E. 292 pages.

Dubois, J. \& F. Dubois-Charlier (inachevé, posthume). Dictionnaire électronique des mots français (DEM), près de 150.000 entrées. Téléchargeable sur :

a) https://www.modyco.fr/fr/base-documentaire/ressources/jean-dubois.html (version Excel)

b) http://rali.iro.umontreal.ca/rali/?q=fr/versions-informatisees-lvf-dem (version

\section{II.2. Articles en collaboration avec Jean Dubois}

Dubois, J. \& F. Dubois-Charlier (1970). «Principes et méthode de l'analyse distributionnelle », in Langages, $n^{\circ} 20$, pp. 3-13.

Dubois, J. \& F. Dubois-Charlier (1973). «Le passif », in Journal de psychologie, $\mathrm{n}^{\circ}$ 2, pp.

Dubois, J. \& F. Dubois-Charlier (1989). « Dictionnaire électronique du verbe français », communication dans Actes du IXè congrès européen sur la grammaire et le lexique des langues

Dubois, J. \& F. Dubois-Charlier (1990). «Incomparabilité des dictionnaires », in Langue française, $\mathrm{n}^{\circ} 87$, pp. 5-10.

Dubois, J. \& F. Dubois-Charlier (1996). « Les collectifs d'êtres vivants », in D. Leeman, \& S. Meleuc (éds) Lexique, syntaxe, traitement automatique. Hommage à Jean Dubois (.), Linx, $\mathrm{n}^{\circ}$ 34, pp. 125-132. Université Paris Ouest Nanterre La Défense.

Dubois, J. \& F. Dubois-Charlier (1997). «Synonymie syntaxique et classification des verbes français ", in Langages, $\mathrm{n}^{\circ} 128$, pp. 51-71.

Dubois, J. \& F. Dubois-Charlier (2010). «La combinatoire lexico-syntaxique dans le Dictionnaire électronique des mots. Les termes de musique à titre d'illustration ", in D. Leeman \& P. Sabatier (éds), Empirie, Théorie, Exploitation: le travail de Jean Dubois sur les verbes français). Langages, $\mathrm{n}^{\circ} 179-180$, pp. 31-56. Paris : Armand Colin.

\section{II.3. Autres ouvrages et articles en collaboration}

Dubois-Charlier, F. et M. Galmiche (1972). "Introduction » de Langages $\mathrm{n}^{\circ} 27$ ( $L a$ sémantique générative), pp. 3-4. Paris : Didier / Larousse.

Anderson J. M, et F. Dubois-Charlier (dir.) (1975). La grammaire des cas, Langages $\mathrm{n}^{\circ} 38$. Paris : Didier / Larousse

Dubois-Charlier, F., Galmiche, M. et M. del Carmen Barrado, (1978 \& 1979), « Semantica generativa ». Madrid: Narcea, Collection Biltacora, 61. 


\section{Larousse}

Comment s'initier à la linguistique, 1 Dialogue préliminaire : les types de phrases Comment s'initier à la linguistique, 2 Les constituants de la phrase Comment s'initier à la linguistique, 3 La représentation graphique de la phrase Comment s'initier à la linguistique, 4 Les transformations de phrase

\section{Traductions}

Gleason H. A. (trad. F. Dubois-Charlier) (1969). Introduction à la linguistique. Paris: Larousse.

Harris, Z. S. (trad. F. Dubois-Charlier) (1969). « Analyse du discours », in Langages, n 13, pp. 8-45. Didier / Larousse.

Kuroda, S. Y. (trad. F. Dubois-Charlier) (1969). «Remarques sur les présuppositions et les contraintes de sélection », in Langages, $\mathrm{n}^{\circ} 14, \mathrm{pp}$. 52-80. Didier / Larousse. 

grammaticale et le rappel immédiat de phrases anglaises ", in Langages, $\mathrm{n}^{\circ} 16$, pp. 91-99. Didier / Larousse. Lyons, J. (trad. F. Dubois-Charlier \& D. Robinson) (1970). Linguistique générale: introduction à la linguistique théorique. Paris : Larousse. Larousse. 